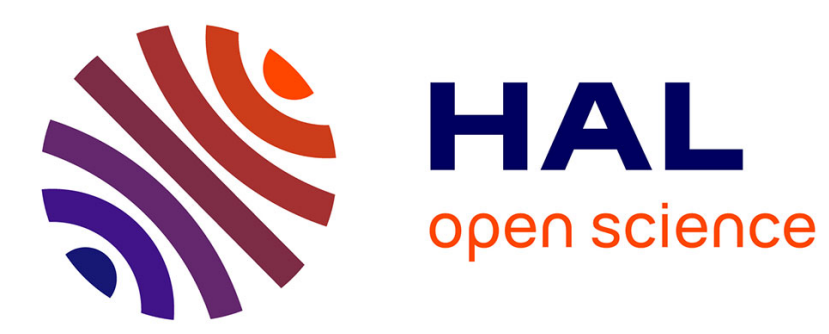

\title{
When is a Matrix of Dimension 3 Similar to a Metzler Matrix? Application to Interval Observer Design
}

Frederic Mazenc, Olivier Bernard

\section{To cite this version:}

Frederic Mazenc, Olivier Bernard. When is a Matrix of Dimension 3 Similar to a Metzler Matrix? Application to Interval Observer Design. IEEE Transactions on Automatic Control, 2021, pp.1-1. 10.1109/TAC.2021.3123245 . hal-03533716

\section{HAL Id: hal-03533716 https://hal.inria.fr/hal-03533716}

Submitted on 21 Jan 2022

HAL is a multi-disciplinary open access archive for the deposit and dissemination of scientific research documents, whether they are published or not. The documents may come from teaching and research institutions in France or abroad, or from public or private research centers.
L'archive ouverte pluridisciplinaire HAL, est destinée au dépôt et à la diffusion de documents scientifiques de niveau recherche, publiés ou non, émanant des établissements d'enseignement et de recherche français ou étrangers, des laboratoires publics ou privés. 


\title{
When is a Matrix of Dimension 3 Similar to a Metzler Matrix?
}

\section{Application to Interval Observer Design}

\author{
Frédéric Mazenc, ${ }^{*} \quad$ Olivier Bernard, ${ }^{\dagger}$
}

\begin{abstract}
A simple necessary and sufficient condition ensuring that a real matrix of dimension 3 is similar to a Metzler matrix is exhibited. When this condition is satisfied, a construction of the transfer matrix is given. This construction is used to design an interval observer for a family of continuous-time systems. An example is provided with interval observer design for the love dynamics in the case of limit cycles.
\end{abstract}

\section{Introduction}

The family of the Metzler matrices play a crucial role in many circumstances, as explained and illustrated for instance in the contributions [15, 7], 9], 10, [16, 1]. In particular, they are crucial in the theory of the positive systems and they can be used to establish stability results for both continuous and discrete-time systems [20, [23], 4], 9, possibly with delay [12], [25]. Moreover, they play a prominent role in the theory of the interval observers, initiated in [11] and developed and applied in recent contributions, as for instance [8, [19, [5] and [2].

However, most of the matrices are not Metzler, which drastically limits the domain of application of the theories based on Metzler matrices. In order to overcome this limitation, in [18], we have shown how, for any matrix $A \in \mathbb{R}^{n \times n}$ with $n \in \mathbb{N}$, one can construct a function $P:[0, \infty) \rightarrow \mathbb{R}^{n \times n}$ of class $C^{1}$, bounded with a bounded inverse and a constant Metzler matrix $M \in \mathbb{R}^{n \times n}$ such that

$$
\dot{P}(t)=M P(t)-P(t) A
$$

for all $t \geq 0$. A constant function $P$ can be found in particular cases, notably when all the eigenvalues of $A$ are real numbers. Indeed, in this case, the Jordan form of the matrix $A$ is Metzler. This function $P$ is especially useful when one wants to transform a linear system into a linear cooperative system: it yields the time-varying change of coordinates $Z=P(t) X$ which transforms the system

$$
\dot{X}(t)=A X(t)
$$

into the cooperative system

$$
\dot{Z}(t)=M Z(t) .
$$

The fundamental message of this result is that any linear time-invariant continuous-time system of any dimension can be transformed into a time-invariant cooperative system. A similar message is given in the paper [19] for discrete-time systems.

Since time-invariant changes of coordinates are simpler than time-varying ones, an interesting question is whether for a given real matrix $A$ there exists a time-invariant change of coordinates which transforms (2) into a cooperative system (3), i.e. whether $A$ is similar to a Metzler matrix $M$.

\footnotetext{
*Inria Saclay, L2S-CNRS-CentraleSupélec, 3 rue Joliot Curie, 91192 Gif-sur-Yvette, France (e-mail: frederic.mazenc@12s.centralesupelec.fr).

${ }^{\dagger}$ INRIA-INRA-CNRS-UPMC, 2004, route des Lucioles - BP 93, 06902 Sophia Antipolis Cedex, France (e-mail: olivier.bernard@inria.fr).
} 
For matrices of dimension 2, an answer to this question is given in the literature: from the main result of the paper [17, one can deduce that a matrix $A \in \mathbb{R}^{2 \times 2}$ is similar to a Metzler matrix if and only if its eigenvalues are real numbers. For matrices of larger dimension, no similar result exists and not many results available in the literature shed light on this problem, even for matrices in $\mathbb{R}^{3 \times 3}$. The most significant one is that some Metzler matrices of dimension 3 have two complex eigenvalues. This is the case for instance of the matrix

$$
U=\left[\begin{array}{lll}
0 & 0 & 1 \\
1 & 0 & 0 \\
0 & 1 & 0
\end{array}\right]
$$

whose eigenvalues are $\left\{1, \frac{-1+i \sqrt{3}}{2}, \frac{-1-i \sqrt{3}}{2}\right\}$.

These remarks motivate the present work. Our aim is to determine a condition ensuring that a matrix in $\mathbb{R}^{3 \times 3}$ is similar to a Metzler matrix. Let us recall that when all the eigenvalues of a matrix of dimension 3 are real numbers, then we know that such a change of coordinates exists since the Jordan form of the matrix is Metzler. We deduce from the properties of $U$ that when 2 of its eigenvalues are not real, then in some cases, such a change of coordinates exists. But it is unknown whether it always exists. We will prove that the answer is no and we will give a necessary and sufficient condition guaranteeing that a matrix $A \in \mathbb{R}^{3 \times 3}$ is similar to a Metzler matrix. This condition can be easily checked and, when it is satisfied, we will provide with the construction of an invertible matrix $P \in \mathbb{R}^{3 \times 3}$ and of a Metzler $M \in \mathbb{R}^{3 \times 3}$ such that $M=P A P^{-1}$. This implies that the result we propose can be straightforwardly used to solve the problems requiring Metzler forms. We also give a necessary and sufficient condition guaranteeing that $A$ is similar to a matrix whose off diagonal entries are positive, which can also be easily checked. Our result complement [6] which proposed a technique making possible for controllable pairs $(A, C)$ to find matrices $P, L$ or appropriate dimension such that $P(A+L C) P^{-1}$ is Metzler.

The paper is organized as follows. Preliminary results are given in Section 2. The main result is stated and proved in Section 3. Section 4 is devoted to an interval observer designed, which uses the main result as key tool. An illustrative example is given in Section 5 . Concluding remarks are drawn in Section 6 .

Notation. We use standard notation, which is simplified when no confusion would arise. The standard Euclidean norm, and the induced matrix norm, are denoted by $|\cdot|$. We let $I$ denote an identity matrix of any dimension. For a matrix $M \in \mathbb{R}^{m \times m}$ whose entries are $m_{i, j} \in \mathbb{R}$, we denote by $M^{+}$the matrix whose entries are $\max \left\{0, m_{i, j}\right\}$ and by $M^{-}$the matrix whose entries are $\max \left\{0,-m_{i, j}\right\}$. For two vectors, $V=\left[v_{1} \ldots v_{r}\right]^{\top} \in \mathbb{R}^{r}$ and $W=\left[w_{1} \ldots w_{r}\right]^{\top} \in \mathbb{R}^{r}$, we write $V \leq W$ when, for all $i \in\{1, \ldots, r\}, v_{i} \leq w_{i}$.

\section{Preliminaries}

\subsection{Perron-Frobenius theorem}

Let us recall a simplified version of Perron-Frobenius theorem [21].

Theorem 1. If $A \in \mathbb{R}^{n \times n}$ is an irreductible nonnegative matrix, then its spectral radius $\mu(A)$ is an eigenvalue of $A$ and there is a vector $V \in \mathbb{R}^{n}$ whose components are all positive such that $A V=\mu(A) V$.

\subsection{Technical results}

In this part, we give first two lemmas which show how, for any matrix in $\mathbb{R}^{3 \times 3}$ with two complex eigenvalues, one can prove through a constructive approach that the matrix is similar to a matrix of the form

$$
\left[\begin{array}{ccc}
\theta & 0 & 0 \\
0 & \kappa & \omega \\
0 & -\omega & \kappa
\end{array}\right] \in \mathbb{R}^{3 \times 3} .
$$

A last lemma, which will be instrumental when we establish the main result, ends the section.

Let us establish a first a result that is devoted to matrices in $\mathbb{R}^{2 \times 2}$. 
Lemma 1. Let us assume that the matrix

$$
G=\left[\begin{array}{ll}
g_{11} & g_{12} \\
g_{21} & g_{22}
\end{array}\right] \in \mathbb{R}^{2 \times 2}
$$

admits 2 complex eigenvalues. Then

$$
\Omega=\sqrt{-g_{12} g_{21}-\left(\frac{g_{11}-g_{11}}{2}\right)^{2}}
$$

is well-defined, positive and the matrix

$$
P=\left[\begin{array}{cc}
\frac{1}{\Omega} & \frac{g_{22}-g_{11}}{2 \Omega g_{21}} \\
0 & -\frac{1}{g_{21}}
\end{array}\right] \in \mathbb{R}^{2 \times 2}
$$

is well-defined, invertible and such that

$$
P G P^{-1}=\left[\begin{array}{cc}
\frac{g_{11}+g_{22}}{2} & \Omega \\
-\Omega & \frac{g_{11}+g_{22}}{2}
\end{array}\right]
$$

Proof. By studying the characteristic polynomial of the matrix $G$, one can prove that this matrix admits two complex eigenvalues if and only if $-g_{12} g_{21}-\left(\frac{g_{11}-g_{11}}{2}\right)^{2}>0$, which implies that $\Omega$ is well-defined and positive. Moreover, the matrix $P$ is well-defined because, due to the fact that no eigenvalue of $G$ is real, $g_{21} \neq 0$. Then through simple calculations, one can check that the equality (8) is satisfied. This concludes the proof.

Let us establish a second a result that is devoted to matrices in $\mathbb{R}^{3 \times 3}$.

Lemma 2. Consider a matrix $A_{0} \in \mathbb{R}^{3 \times 3}$ which admits complex eigenvalues. Then there are real numbers $\theta, \kappa$ and $\omega>0$ such that the spectrum of $A_{0}$ is $\{\theta, \kappa+i \omega, \kappa-i \omega\}$ and there is an invertible matrix $Q \in \mathbb{R}^{3 \times 3}$ such that

$$
Q A_{0} Q^{-1}=A_{1}
$$

with

$$
A_{1}=\left[\begin{array}{ccc}
\theta & 0 & 0 \\
0 & \kappa & \omega \\
0 & -\omega & \kappa
\end{array}\right] \in \mathbb{R}^{3 \times 3} .
$$

Proof. We deduce from [13, p. 73] that $A_{0}$ is similar to the matrix $A_{1}$. One can determine an explicit expression for $Q$ by putting first $A$ in a triangular form with a second diagonal block of dimension 2 and next by taking advantage of Lemma 1 .

The last result of the section is the following:

Lemma 3. Consider a matrix

$$
A_{1}=\left[\begin{array}{ccc}
\theta & 0 & 0 \\
0 & \kappa & \omega \\
0 & -\omega & \kappa
\end{array}\right] \in \mathbb{R}^{3 \times 3}
$$

with $\theta \in \mathbb{R}, \kappa \in \mathbb{R}$ and $\omega>0$. Let

$$
A_{2}=\left[\begin{array}{ccc}
\frac{\theta-\kappa}{\omega} & 0 & 0 \\
0 & 0 & 1 \\
0 & -1 & 0
\end{array}\right]
$$

Let $P \in \mathbb{R}^{3 \times 3}$ be an invertible matrix. Then $P A_{1} P^{-1}$ a Metzler matrix if and only if $P A_{2} P^{-1}$ is a Metzler matrix. 
Proof. Since

$$
A_{1}=\left[\begin{array}{ccc}
\theta-\kappa & 0 & 0 \\
0 & 0 & \omega \\
0 & -\omega & 0
\end{array}\right]+\kappa I=\omega A_{2}+\kappa I
$$

and $\omega>0$, then

$$
P A_{1} P^{-1}=\omega P A_{2} P^{-1}+\kappa I .
$$

This allows us to conclude.

\section{Main result}

In this section, we establish the main theoretical result of the work:

Theorem 2. Let $A \in \mathbb{R}^{3 \times 3}$ be a matrix whose spectrum is $\{\theta, \kappa+i \omega, \kappa-i \omega\}$ with $\theta \in \mathbb{R}, \kappa \in \mathbb{R}$ and $\omega>0$. This matrix is similar to a Metzler matrix if and only if the inequality

$$
\theta \geq \kappa+\sqrt{3} \omega
$$

is satisfied. When (15) is satisfied, this matrix is similar to the Metzler matrix:

$$
H=\frac{1}{3}\left[\begin{array}{ccc}
\theta+2 \kappa & \theta-\kappa-\sqrt{3} \omega & \theta-\kappa+\sqrt{3} \omega \\
\theta-\kappa+\sqrt{3} \omega & \theta+2 \kappa & \theta-\kappa-\sqrt{3} \omega \\
\theta-\kappa-\sqrt{3} \omega & \theta-\kappa+\sqrt{3} \omega & \theta+2 \kappa
\end{array}\right] .
$$

The matrix $A$ is similar to a matrix whose off-diagonal entries are positive if and only if

$$
\theta>\kappa+\sqrt{3} \omega .
$$

Remark. When the inequality (17) is satisfied, then all the off diagonal entries of $H$ are positive.

\subsection{Proof of Theorem 2}

\subsubsection{Preliminary remarks}

Since $A \in \mathbb{R}^{3 \times 3}$ is a matrix whose spectrum is $\{\theta, \kappa+i \omega, \kappa-i \omega\}$, then, according to Lemma 2 it is similar to the matrix:

$$
A_{1}=\left[\begin{array}{ccc}
\theta & 0 & 0 \\
0 & \kappa & \omega \\
0 & -\omega & \kappa
\end{array}\right] .
$$

Next, Lemma 3 prompts us to study first the matrix:

$$
A_{\sharp}=\left[\begin{array}{ccc}
\alpha & 0 & 0 \\
0 & 0 & 1 \\
0 & -1 & 0
\end{array}\right]
$$

with

$$
\alpha=\frac{\theta-\kappa}{\omega} .
$$

\subsubsection{First part}

In this part, we prove the following result:

Proposition 1. The matrix $A_{1}$ defined in (18) is not similar to a Metzler matrix when the inequality

$$
\theta<\sqrt{3} \omega+\kappa
$$

is satisfied. 
Proof. We distinguish between 2 cases.

First case: $\theta \leq \kappa$. Then $\alpha$ defined in 200 satisfies $\alpha \leq 0$.

Let us proceed by contradiction: let us assume that $A_{\sharp}$ defined in $(19)$ is similar to a Metzler matrix $M \in \mathbb{R}^{3 \times 3}$.

Then there is a constant $d \in \mathbb{R}$ such that the matrix

$$
N=d I+M
$$

is nonnegative, irreductible and with positive diagonal entries. Indeed, if it was reductible, a permutation would yield a block diagonal matrix with a block of dimension 2 associated to complex eigenvalues, which would not be nonnegative [17]. Then Perron-Frobenius theorem ensures that the spectral radius of $N$, denoted $\mu(N)$, is an eigenvalue of $N$ and $\mu(N) \geq 0$.

Now, observe that the eigenvalues of the matrix $N$ are $d+\alpha, d+i$ and $d-i$ because the eigenvalues of $A_{\sharp}$ are $\alpha, i,-i$. As an immediate consequence,

$$
\mu(N)=d+\alpha
$$

because $d+\alpha$ is the unique eigenvalue of $N$ that is a real number. On the other hand, by definition,

$$
\mu(N)=\max \left\{|d+\alpha|, \sqrt{1+d^{2}}\right\} .
$$

It follows that

$$
d+\alpha=\max \left\{d+\alpha, \sqrt{1+d^{2}}\right\} \geq \sqrt{1+d^{2}} .
$$

As an immediate consequence,

$$
\alpha \geq \sqrt{1+d^{2}}-d>0
$$

which is in contradiction with $\alpha \leq 0$.

Second case: $\theta \in(\kappa, \kappa+\sqrt{3} \omega)$. Then $\alpha$ defined in 20$)$ satisfies $\alpha \in(0, \sqrt{3})$.

Let us proceed by contradiction: let us assume that $A_{\sharp}$ in 19$)$ is similar to a Metzler matrix $M=\left(m_{i j}\right)$ i.e. there is an invertible matrix $P \in \mathbb{R}^{3 \times 3}$ such that $P A_{\sharp} P^{-1}=M$. It follows that

$$
P A_{\sharp}^{2} P^{-1}=M^{2} .
$$

Since

$$
A_{\sharp}^{2}=\left[\begin{array}{ccl}
\alpha^{2} & 0 & 0 \\
0 & -1 & 0 \\
0 & 0 & -1
\end{array}\right],
$$

it follows that the trace of $A_{\sharp}^{2}$ is $\alpha^{2}-2$. On the other hand, the trace of $M^{2}$ is

$$
m_{11}^{2}+m_{22}^{2}+m_{33}^{2}+2 m_{12} m_{21}+2 m_{13} m_{31}+2 m_{23} m_{32} .
$$

Since the trace of $A_{\sharp}^{2}$ is equal to the trace of $M^{2}$, the equality

$$
\alpha^{2}=m_{11}^{2}+m_{22}^{2}+m_{33}^{2}+2 m_{12} m_{21}+2 m_{13} m_{31}+2 m_{23} m_{32}+2
$$

is satisfied. Since $M$ is Metzler, $2 m_{12} m_{21}+2 m_{13} m_{31}+2 m_{23} m_{32} \geq 0$, which, in combination with (30), implies that

$$
\alpha^{2} \geq m_{11}^{2}+m_{22}^{2}+m_{33}^{2}+2
$$

On the other hand, the trace of $M$ is equal to the trace of $A_{\sharp}$, which implies that

$$
m_{33}=\alpha-\left(m_{11}+m_{22}\right) \text {. }
$$


By combining (31) and 32 , we obtain

$$
\alpha^{2} \geq m_{11}^{2}+m_{22}^{2}+\left(\alpha-m_{11}-m_{22}\right)^{2}+2 .
$$

This inequality is equivalent to

$$
\alpha\left(m_{11}+m_{22}\right) \geq m_{11}^{2}+m_{22}^{2}+m_{11} m_{22}+1 .
$$

Therefore $m_{11}+m_{22}>0$ and

$$
\alpha \geq \Gamma\left(m_{11}, m_{22}\right)
$$

with

$$
\Gamma\left(m_{11}, m_{22}\right)=\frac{m_{11}^{2}+m_{22}^{2}+m_{11} m_{22}+1}{m_{11}+m_{22}} .
$$

Now, let us study $\Gamma$. If $m_{11} m_{22} \leq 0$, then

$$
\Gamma\left(m_{11}, m_{22}\right)=m_{11}+m_{22}+\frac{1-m_{11} m_{22}}{m_{11}+m_{22}} \geq m_{11}+m_{22}+\frac{1}{m_{11}+m_{22}} \geq 2 .
$$

Now, consider the case where $m_{11}>0$ and $m_{22}>0$. From $\Gamma(\ell, 0)=\ell+\frac{1}{\ell} \geq 2$ and $\Gamma(0, \ell) \geq 2$ for all $\ell>0$, we deduce that the smallest value taken by $\Gamma$ in $(0,+\infty) \times(0,+\infty)$ is either 2 or $\Gamma\left(m_{11, \star}, m_{22, \star}\right)$ where $\left(m_{11, \star}, m_{22, \star}\right) \in(0,+\infty) \times(0,+\infty)$ is such that

$$
\frac{\partial \Gamma}{\partial m_{11}}\left(m_{11, \star}, m_{22, \star}\right)=\frac{\partial \Gamma}{\partial m_{22}}\left(m_{11, \star}, m_{22, \star}\right)=0 .
$$

Simple calculations give

$$
\frac{\partial \Gamma}{\partial m_{11}}\left(m_{11}, m_{22}\right)=\frac{2 m_{11}+m_{22}}{m_{11}+m_{22}}-\frac{m_{11}^{2}+m_{22}^{2}+m_{11} m_{22}+1}{\left(m_{11}+m_{22}\right)^{2}}
$$

and

$$
\frac{\partial \Gamma}{\partial m_{22}}\left(m_{11}, m_{22}\right)=\frac{m_{11}+2 m_{22}}{m_{11}+m_{22}}-\frac{m_{11}^{2}+m_{22}^{2}+m_{11} m_{22}+1}{\left(m_{11}+m_{22}\right)^{2}} .
$$

These equalities and (38) imply that $m_{11, \star}=m_{22, \star}$. This equality and 390 and 40 give:

$$
\frac{\partial \Gamma}{\partial m_{11}}\left(m_{11, \star}, m_{22, \star}\right)=\frac{3}{2}-\frac{3 m_{11, \star}^{2}+1}{4 m_{11, \star}^{2}}=\frac{\partial \Gamma}{\partial m_{22}}\left(m_{11, \star}, m_{22, \star}\right) .
$$

Thus

$$
\frac{\partial \Gamma}{\partial m_{11}}\left(m_{11, \star}, m_{11, \star}\right)=\frac{3}{4}-\frac{1}{4 m_{11, \star}^{2}} .
$$

Then the equality $\frac{\partial \Gamma}{\partial m_{11}}\left(m_{11, \star}, m_{11, \star}\right)=0$ implies that $m_{11, \star}=\frac{1}{\sqrt{3}}$. Consequently, $\left(m_{11, \star}, m_{22, \star}\right)=$ $\left(\frac{1}{\sqrt{3}}, \frac{1}{\sqrt{3}}\right)$. We deduce that when $m_{11}>0$ and $m_{22}>0$, then

$$
\Gamma\left(m_{11}, m_{22}\right) \geq \Gamma\left(m_{11, \star}, m_{22, \star}\right)=\frac{\frac{1}{3}+\frac{1}{3}+\frac{1}{3}+1}{2 \frac{1}{\sqrt{3}}}=\sqrt{3} .
$$

Since $\alpha<\sqrt{3}$, it follows that 35 is not satisfied. This yields a contradiction.

\subsubsection{Second part}

Let us introduce a matrix

$$
R=\left[\begin{array}{ccc}
1 & 0 & 2 \\
1 & \sqrt{3} & -1 \\
1 & -\sqrt{3} & -1
\end{array}\right]
$$

and prove the following result: 
Proposition 2. The matrix $A_{1}$ defined in $(18)$ is similar to a Metzler matrix when

$$
\theta \geq \kappa+\sqrt{3} \omega
$$

The equality

$$
R A_{1} R^{-1}=H
$$

with $R$ defined in (44) is satisfied and $H$ defined in (16).

Proof. One can prove the result through simple calculations. For instance, one can show that

$$
R A_{1}=H R=\left[\begin{array}{ccc}
\theta & -2 \omega & 2 \kappa \\
\theta & \sqrt{3} \kappa+\omega & \sqrt{3} \omega-\kappa \\
\theta & \omega-\sqrt{3} \kappa & -\sqrt{3} \omega-\kappa
\end{array}\right] .
$$

\subsubsection{Conclusion of the proof}

From Lemma 2, we deduce that the matrix $A$ is similar to a Metzler matrix if and only if the matrix $A_{1}$ defined in (18) with $\omega>0$ is similar to a Metzler matrix. Then, from the first and the second part of the proof of Theorem 2, we can easily complete the proof, except that we must still show that the matrix $A$ is similar to a matrix whose off-diagonal entries are positive if and only if (17) holds. The sufficient part of this assertion follows from the off-diagonal entries of $H$ being positive when (17) holds. Nothing has to be proved in the case where $\theta<\kappa+\sqrt{3} \omega$ because the second part of the proof of Theorem 2 ensures that $A$ is not similar to a Metzler matrix. Thus, what remains to be studied is the limit case: $\theta=\kappa+\sqrt{3} \omega$.

Let us proceed by contradiction. Let us assume that $\theta=\kappa+\sqrt{3} \omega$ and $A_{1}$ is similar to a matrix $F$ whose off-diagonal entries are positive. Then there is a constant $\bar{\epsilon}>0$ such that this property holds for any matrix $F_{\ddagger} \in \mathbb{R}^{3 \times 3}$ such that $\left|F-F_{\ddagger}\right| \leq \bar{\epsilon}$. By continuity of the eigenvalues of a matrix with respect to its entries, we deduce that there are constants $\theta, \kappa$ and $\omega$ such that $\theta<\kappa+\sqrt{3} \omega$ and the corresponding matrix $A_{1, \triangle}$ is similar to a matrix $F_{\triangle}$ such that $\left|F-F_{\triangle}\right| \leq \bar{\epsilon}$. It follows that $A_{1, \triangle}$ is similar to a matrix whose off-diagonal entries are positive. This yields a contradiction with the fact that $\theta<\kappa+\sqrt{3} \omega$. We conclude that $A_{1}$ is not similar to a Metzler matrix.

\section{Application to interval observer design}

In this section, we take advantage of Theorem 2 to construct time-invariant interval observers for a family of systems which is frequently encountered in practice. Throughout this section, we use the notation introduced in the previous sections.

We consider the system:

$$
\dot{x}(t)=\left(A_{1}+B\right) x(t)+w+\lambda(t)
$$

with $x \in \mathbb{R}^{3}$, where $A_{1} \in \mathbb{R}^{3 \times 3}$ is the matrix defined in $100, B \in \mathbb{R}^{3 \times 3}$, w is a constant vector and $\lambda:[0,+\infty) \rightarrow \mathbb{R}^{3}$ is a continuous function. Let us observe that $\Lambda$ may represent a measured nonlinear term that depends on $x(t)$.

Let us introduce two assumptions:

Assumption A. The eigenvalues of $A$ are $\theta, \kappa+i \omega, \kappa-i \omega$ and the inequality $\theta>\sqrt{3} \omega+\kappa$ holds. The entries of the matrix $B_{\dagger}=R B R^{-1}=\left(b_{i, j \dagger}\right)$ satisfy:

$$
\begin{array}{ll}
b_{i, j \dagger} \geq \kappa+\sqrt{3} \omega-\theta, & \forall(i, j) \in\{(1,2),(2,3),(3,1)\} \\
b_{i, j \dagger} \geq \kappa-\sqrt{3} \omega-\theta
\end{array}, \quad \forall(i, j) \in\{(1,3),(2,1),(3,2)\} .
$$

Assumption B. Two vectors $\bar{w} \in \mathbb{R}^{3}$ and $\underline{w} \in \mathbb{R}^{3}$ and two continuous functions $\bar{\lambda}:[0,+\infty) \rightarrow \mathbb{R}^{3}$ and $\underline{\lambda}:[0,+\infty) \rightarrow \mathbb{R}^{3}$ such that

$$
\underline{w} \leq w \leq \bar{w}
$$


and

are known.

$$
\underline{\lambda}(t) \leq \lambda(t) \leq \bar{\lambda}(t) \quad, \quad \forall t \geq 0
$$

We are ready to state and prove the following result:

Proposition 3. Let the system (48) satisfy Assumptions $A$ and B. Then

$$
\left\{\begin{aligned}
\dot{\bar{z}}(t) & =\left(H+R B R^{-1}\right) \bar{z}(t)+R^{+} \bar{w}-R^{-} \underline{w}+R^{+} \bar{\lambda}(t)-R^{-} \underline{\lambda}(t) \\
\dot{z}(t) & =\left(H+R B R^{-1}\right) \underline{z}(t)+R^{+} \underline{w}-R^{-} \bar{w}+R^{+} \underline{\lambda}(t)-R^{-} \overline{\bar{\lambda}}(t) \\
\bar{x}(t) & =\left(R^{-1}\right)^{+} \bar{z}(t)-\left(R^{-1}\right)^{-} \underline{z}(t) \\
\underline{x}(t) & =\left(R^{-1}\right)^{+} \underline{z}(t)-\left(R^{-1}\right)^{-} \bar{z}(t) \\
\bar{z}\left(t_{0}\right) & =R^{+} x_{l}\left(t_{0}\right)-R^{-} x_{s}\left(t_{0}\right) \\
\underline{z}\left(t_{0}\right) & =R^{+} x_{s}\left(t_{0}\right)-R^{-} x_{l}\left(t_{0}\right),
\end{aligned}\right.
$$

where $H$ is the matrix defined in (16) and $R$ is the matrix defined in (44), is an interval observer for the system (48), i.e. if the inequalities $x_{s}\left(t_{0}\right) \leq x\left(t_{0}\right) \leq x_{l}\left(t_{0}\right)$ hold, then the inequalities $\underline{x}(t) \leq x(t) \leq \bar{x}(t)$ hold for all $t \geq t_{0}$.

Proof. According to Proposition 2, the change of coordinates $z(t)=R x(t)$ gives:

$$
\dot{z}(t)=\left(R A_{1} R^{-1}+R B R^{-1}\right) z(t)+R w+R \Lambda(t) .
$$

This systems can be rewritten as:

$$
\dot{z}(t)=\left(H+B_{\dagger}\right) z(t)+R w+R \Lambda(t),
$$

where $B_{\dagger}=R B R^{-1}$. Assumption A ensures that the matrix $H+B_{\dagger}$ is Metzler. This fact, in combination with Assumption B, ensures that

$$
\left\{\begin{array}{l}
\dot{\bar{z}}=\left(H+B_{\dagger}\right) \bar{z}(t)+R^{+} \bar{w}-R^{-} \underline{w}+R^{+} \bar{\lambda}(t)-R^{-}-\bar{\lambda}(t) \\
\dot{z}=\left(H+B_{\dagger}\right) \underline{z}(t)+R^{+} \underline{w}-R^{-} \bar{w}+R^{+} \underline{\lambda}(t)-R^{-} \overline{\bar{\lambda}}(t)
\end{array}\right.
$$

with $\bar{z} \in \mathbb{R}^{3}$ and $\underline{z} \in \mathbb{R}^{3}$, is such that for initial conditions satisfying the inequalities

$$
\underline{z}\left(t_{0}\right) \leq z\left(t_{0}\right) \leq \bar{z}\left(t_{0}\right)
$$

then

$$
\underline{z}(t) \leq z(t) \leq \bar{z}(t)
$$

for all $t \geq t_{0}$. Then arguing for instance as in the proof of [22, Theorem 4], we can conclude.

\section{Example: the dynamics of love}

\subsection{Case study presentation}

S. Rinaldi 24 proposed a model describing the love dynamics between Laura de Noves and Petrarch, demonstrating that it leads to periodic dynamics. One of the most interesting point of this work is that the model was calibrated and validated using data. The 23 dated poems out of the 366 poems Petrarch wrote in the Canzoniere during 21 years were analyzed from a linguistic and lyric point of view 14. A grade $P \in(-1 ; 1)$ was then assigned to each poem. The maximum grade corresponds to ecstatic love, and the minimum stands for deep despair. There is obviously an uncertainty inherent to the quantitative estimate of Petrarch's mood, but also there are uncertainties in the dating of some of the poems.

On this basis, Rinaldi's model describes the love dynamics by three variables. The love of Laura for Petrarch is denoted $L$. The love of Petrarch for Laura $(P)$ is also related to its poetic inspiration $Z$. The model writes as follows:

$$
\left\{\begin{aligned}
\dot{L}(t) & =-\alpha_{1} L(t)+R_{L}(P(t))+A_{p} \\
\dot{P}(t) & =-\alpha_{2} P(t)+R_{P}(L(t))+\beta_{2} A_{L}(Z(t)) \\
\dot{Z}(t) & =-\alpha_{3} Z(t)+\beta_{4} P(t)
\end{aligned}\right.
$$


The positive real numbers $\alpha_{i}, i=1,2,3$ represent the sentiment forgetting rate for each individual and for Petrarch's inspiration. The terms $R_{L}(P)$ (resp. $R_{P}(L)$ ) is the reaction function, which quantifies the reaction of Laura (resp. Petrarch) to Petrarch's (resp. Laura's) love. The term $A_{P}\left(\right.$ resp. $\left.A_{L}(Z)\right)$ is the appeal of Laura (resp. Petrarch) for Petrarch (resp. Laura). The function $R_{P}(L)$ is linear while the nonlinear reaction function $R_{L}(P)$ accounts for the fact that Laura feels very sorry for Petrarch when he is crossing a despairing phase:

$$
R_{L}(P)=\beta_{1} P\left(1-\left(\frac{P}{\gamma}\right)^{2}\right) \quad, \quad R_{P}(L)=\beta_{4} L .
$$

Finally, the decreasing function $A_{L}(Z)$ represents the fact that artistic inspiration "attenuates the role of the most basic instincts". Rinaldi proposed a hyperbolic term, but we choose a simpler linear function:

$$
A_{L}(Z)=\beta_{2}-\beta_{3} Z
$$

The term $A_{P}$ is constant $\left(A_{P}=\beta_{1}\right)$.

In this example, it is assumed that the reaction function of Laura for Petrarch can be evaluated from her social behaviour, i.e. from her mood (see Figure $1 \mathrm{~b}$ ).

All the model parameters are positive. They were recalibrated to fit the available data (see Table 1), as shown in Figure 1;). A limit cycle is rapidly reached as shown on figure 1 a.

\begin{tabular}{|lc|lc|}
\hline Parameter & value & Parameter & value \\
\hline$\alpha_{1}$ & 4.8 & $\beta_{1}$ & 21.3 \\
$\alpha_{2}$ & 77.76 & $\beta_{2}$ & 333 \\
$\alpha_{3}$ & 4.096 & $\beta_{3}$ & 29.4048 \\
$\gamma$ & 0.4800 & $\beta_{4}$ & 0.4500 \\
\hline
\end{tabular}

Table 1: Parameters used to simulate the love model (a recalibration was carried out to account for the slightly different model compared to [24].)

Now the love model takes the following form:

$$
\dot{x}(t)=A_{0} x(t)+b+\psi(t)
$$

where

$$
A_{0}=\left[\begin{array}{ccc}
-\alpha_{1} & 0 & 0 \\
0 & -\alpha_{2} & -\beta_{3} \\
0 & \beta_{4} & -\alpha_{3}
\end{array}\right] ; b=\left[\begin{array}{c}
\beta_{1} \\
\beta_{2} \\
0
\end{array}\right] .
$$

The vector $\psi(t)=\left[\begin{array}{lll}R_{L}(P(t)) & 0 & 0\end{array}\right]^{\top}$ contains Laura's mood, supposed to be the measured output. The objective is now to design an interval observer so as to reconstruct the love of Laura and Petrarch, together with the inspiration of Petrarch from $\psi(t)$. There is obviously an uncertainty in the interpretation of the mood of Laura, and therefore we assume that:

$$
\underline{\psi}(t) \leq \psi(t) \leq \bar{\psi}(t)
$$

for all $t \geq 0$ where $\bar{\psi}$ and $\underline{\psi}$ are two known continuous functions. In the same way, we assume uncertainty on the vector $b$ :

$$
\underline{b} \leq b \leq \bar{b},
$$

where $\bar{b} \in \mathbb{R}^{3}$ and $\underline{b} \in \mathbb{R}^{3}$ are two known vectors.

\subsection{Observer design}

Let $Q$ denote a matrix such that $Q A_{0} Q^{-1}=A_{1}$, where $A_{0}$ is the matrix defined in $(62)$ and $A_{1}$ is the matrix in the canonical form (47). (Let us recall that $Q$ can be constructed using Lemma 1). Since, with the chosen parameter values, the spectrum of $A_{1}$ is $\{-4.8000 ;-14.9424+3.4674 i ;-14.9424-3.4674 i\}$, one can check easily that that the necessary and sufficient condition of Theorem 2 is satisfied:

$$
-4.8000 \geq-14.9424+3.4674 \sqrt{3} .
$$


As a consequence $A_{1}$ is similar to a Metzler with $R$ given by (44) as transfer matrix.

Proposition 3 gives us the interval observer:

$$
\left\{\begin{aligned}
\dot{\bar{z}}(t) & =H \bar{z}(t)+\bar{b}_{H}+\bar{\psi}_{H}(t) \\
\dot{z}(t) & =H \underline{z}(t)+\underline{b}_{H}+\underline{\psi}_{H}(t) \\
\bar{x}(t) & =\left((R Q)^{-1}\right)^{+} \bar{z}(t)-\left((R Q)^{-1}\right)^{-} \underline{z}(t) \\
\underline{x}(t) & =\left((R Q)^{-1}\right)^{+} \underline{z}(t)-\left((R Q)^{-1}\right)^{-} \bar{z}(t) \\
\underline{z}\left(t_{0}\right) & =(R Q)^{+} x_{l}\left(t_{0}\right)-(R Q)^{-} x_{s}\left(t_{0}\right) \\
\bar{z}\left(t_{0}\right) & =(R Q)^{+} x_{s}\left(t_{0}\right)-(R Q)^{-} x_{l}\left(t_{0}\right),
\end{aligned}\right.
$$

with

$$
\begin{aligned}
\underline{b}_{H} & =(R Q)^{+} \underline{b}-(R Q)^{-} \bar{b}, \\
\bar{b}_{H} & =(R Q)^{+} \overline{\bar{b}}-(R Q)^{-} \underline{\underline{b}}, \\
\bar{\psi}_{H}(t) & =(R Q)^{+} \bar{\psi}(t)-(R Q)^{-} \underline{\psi}(t), \\
\underline{\psi}_{H}(t) & =(R Q)^{+} \underline{\psi}(t)-(R Q)^{-} \overline{\bar{\psi}}(t) .
\end{aligned}
$$

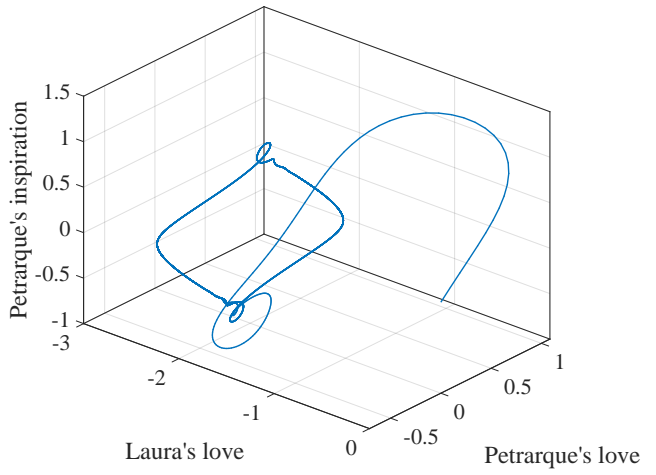

(a) Trajectory reaching the limit cycle

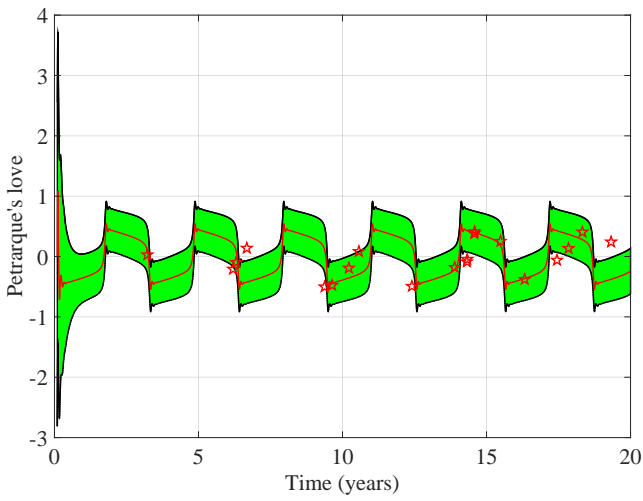

(c) Interval estimation of Petrarque's love and experimental data points $(\star)$

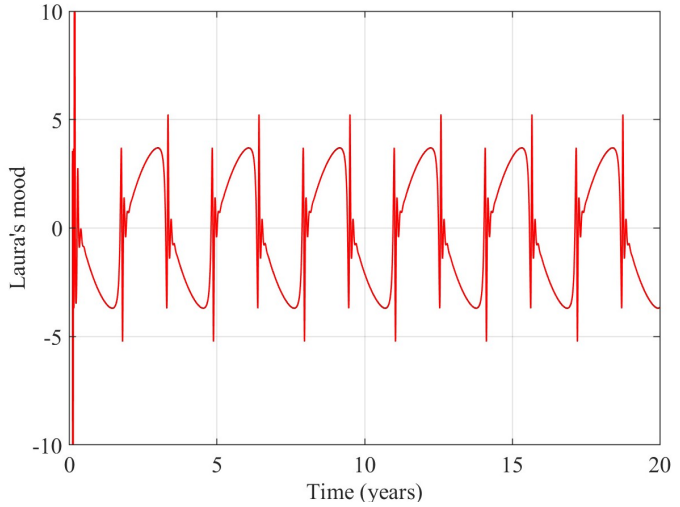

(b) Laura's mood

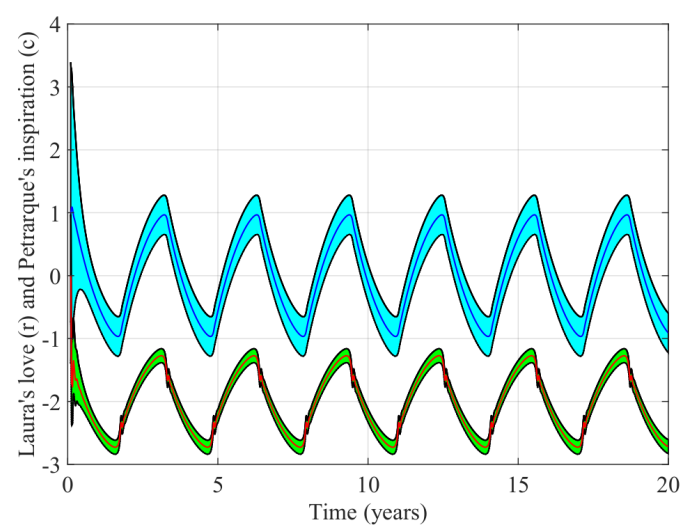

(d) Interval estimate of variables $L$ (green) and $Z$ (blue)

Figure 1: Simulation of the love model and interval observer estimation.

The observer is run on Figure 1k, assuming that the initial conditions are unknown but with known bounds. Laura's mood as measured in Figure 1 $\mathrm{b}$ was used by the interval observer. The interval predictions encompass the data points estimated from the analysis of Petrarque's sonnets. The state interval prediction is also presented on Figure 1 . 


\section{Conclusion}

For the real square matrices of dimension 3, we determined a necessary and sufficient condition ensuring that a matrix is similar to a Metzler matrix. For matrices satisfying this condition, we exhibit a transfer matrix. We have used it to build interval observers for a family of continuous-time systems with uncertain disturbances.

Extensions of the main result of our work to matrices of dimension larger than 3 are expected and will be the subject of further studies as long as their application to interval observer designs.

\section{References}

[1] M. Ait Rami, Stability analysis ans synthesis for linear positive systems with time-varying delays. In Lecture notes in control and information sciences, Vol. 289, pp. 205-215. Berlin: Springer-Verlag.

[2] M. Ait Rami, M. Schonlein, J. Jordan, Estimation of Linear Positive Systems with Unknown TimeVarying Delays. Europ. Jour. of Control, 19 (3), pp. 179-187, May 2013.

[3] A. Berman, R. J. Plemmons, Nonnegative Matrices in the Mathematical Sciences. New York: Academic Press, 1979.

[4] F. Blanchini, P. Colaneri, M. E. Valcher, Switched Positive Linear Systems. Foundations and Trends in Systems and Control 2 (2), pp. 101-273, 2015.

[5] F. Cacace, A. Germani, C. Manes, A new approach to design interval observers for linear systems. IEEE Trans. Aut. Control, 60(6), April 2015.

[6] E. Chambon, P. Apkarian, L. Burlion, Metzler Matrix Transform Determination using a Nonsmooth Optimization Technique with an Application to Interval Observers. SIAM Conference on Control and its Applications, Paris, 2015.

[7] S. Dashkovskiy, B. Ruffer, F. Wirth, An ISS small-gain theorem for general networks. Mathematics of Control, Signals, and Systems, 19, pp. 93-122, 2007.

[8] D. Efimov, T. Raissi, Design of interval observers for uncertain dynamical systems. Automation and Remote Control, vol. 77, pp.-225 (2016)

[9] E. Fornasini, M. E. Valcher, Linear copositive Lyapunov functions for continuous-time positive switched systems. IEEE Trans. Aut. Control, vol. 55, No. 8, pp. 1933-1937, 2010.

[10] E. Fornasini, M. E. Valcher, Stability and Stabilizability Criteria for Discrete-Time Positive Switched Systems. IEEE Trans. Aut. Control, vol. 57, No. 5, May 2012.

[11] J.-L. Gouzé, A. Rapaport, Z. Hadj-Sadok, Interval observers for uncertain biological systems. Ecological Modelling, 133, pp. 45-56, 2000.

[12] W. M. Haddad, V. Chellaboina, Q. Hui, Nonnegative and Compartmental Dynamical Systems. Princeton University Press, Princeton and Oxford, 2010.

[13] S. B. Hsu, Ordinary differential equations with applications. Series on Applied Mathematics, Volume $16,2005$.

[14] F. J. Jones. The Structure of Petrarch's Canzoniere: A Chronological, Psychological, and Stylistic Analysis. Boydell \& Brewer; 1995.

[15] M. Kieffer, E. Walter, Guaranteed nonlinear state estimator for cooperative systems. Numerical Algorithms, 37, 1, pp. 187-198, 2004.

[16] O. Mason, R. N. Shorten, On linear copositive Lyapunov functions and the stability of switched positive linear systems, IEEE Trans. Aut. Control, vol. 52, no. 7, pp. 1346-1349, 2007. 
[17] F. Mazenc, O. Bernard, Asymptotically Stable Interval Observers for Planar Systems with Complex Poles, IEEE Trans. Aut. Control, vol. 55, Issue 2, pp. 523-527, Feb. 2010.

[18] F. Mazenc, O. Bernard, Interval observers for linear time-invariant systems with disturbances. Automatica, 47 (1), pp. 140-147, 2011.

[19] F. Mazenc, T. N. Dinh, S.I. Niculescu, Interval Observers for Discrete-time Systems, International Journal of Robust and Nonlinear Control, 24: pp. 2867-2890, 2014.

[20] F. Mazenc, M. Malisoff, Stability Analysis for Time-Varying Systems with Delay using Linear Lyapunov Functionals and a Positive Systems Approach, IEEE Trans. Aut. Control, vol. 61, No. 3, pp. 771-776, 2016.

[21] P. Ngoc, A Perron-Frobenius theorem for a class of positive quasi-polynomial matrices. Applied Mathematics Letters, 19, pp. 747-751, 2006.

[22] T. Raissi, D. Efimov, A. Zolghadri, Interval State Estimation for a Class of Nonlinear Systems. IEEE Trans. On Aut. Contr., Vol. 57, No. 1, Jan 2012.

[23] A. Rantzer, Distributed control of positive systems. European Journal of Control, 24, pp. 72-80, 2015.

[24] S. Rinaldi, Laura and Petrarch: An intriguing case of cyclical love dynamics. SIAM Journal on Applied Mathematics. 58(4), vol.1205-21, 1998.

[25] L. Farina, S. Rinaldi, Positive Linear Systems: Theory and Applications. New York: Wiley-Interscience, 2011, Series on Pure and Applied Mathematics. 